\title{
ARRB2 Gene
}

National Cancer Institute

\section{Source}

National Cancer Institute. ARRB2 Gene. NCI Thesaurus. Code C129045.

This gene is involved in receptor desensitization. 